\title{
Monoclonal Antibody 48.7
}

National Cancer Institute

\section{Source}

National Cancer Institute. Monoclonal Antibody 48.7. NCI Thesaurus. Code C29199.

A mouse monoclonal antibody (MoAb) with proteoglycan antigen detection and binding activity. Hybridomas of SP2/0 mouse myeloma cells and mouse spleen cells are immunized with cultured human melanoma cells, and subsequently one of the hybridomas secreted MoAb 48.7. MoAb 48.7 is used for detection and binding to the proteoglycan antigen expressed on the cell surface of melanoma cells. 